\title{
INGESTÃO DE FIBRA ALIMENTAR E TEMPO DE TRÂNSITO COLÔNICO EM PACIENTES COM CONSTIPAÇÃO FUNCIONAL
}

\author{
Adriana Cruz LOPES ${ }^{1}$ e Carlos Roberto VICTORIA ${ }^{2}$
}

RESUMO - Racional - Pacientes com constipação funcional que não melhoram com suplementação de fibras dietéticas, representam importante problema clínico. Objetivos - Avaliar as relações entre as quantidades de fibras ingeridas, intensidade da constipação e o tempo de trânsito colônico em pacientes com constipação funcional. Métodos - Foram avaliados 30 pacientes constipados funcionais, sem melhoras após suplementação dietética com fibras e 18 pessoas controle, sadias, sem queixas digestivas, utilizando inquéritos individuais quanto à ingestão de fibras e a intensidade da constipação e, por meio da técnica dos marcadores radiopaco, o tempo de trânsito colônico, total e segmentar. Resultados - Apesar da maior ingestão diária de fibras (26,3 $\pm 12,9 \mathrm{~g}$, constipados x 9,3 $\pm 5,2 \mathrm{~g}$, controles), os sintomas da constipação mostraram-se intensos nos constipados (escore médio $=21,3 \pm 4,07$ ). O tempo normal para o trânsito colônico foi de 58,8h. O trânsito colônico total, em média, foi mais lento nos constipados ( $41,0 \pm 22,8 \mathrm{~h}$, constipados x $21,8 \pm 18,5 \mathrm{~h}$, controles). Constipados com trânsito lento ( $>58,8 \mathrm{~h}$ ) apresentaram inércia colônica (oito), obstrução de saída (um) e lentificação no cólon esquerdo (um). Constipados com trânsito normal ( $<58,8 \mathrm{~h}$ ), apresentaram lentificações isoladas no cólon direito (nove), no cólon esquerdo (três) e no segmento retossigmoideano (oito). Não houve correlação entre a quantidade ingerida de fibra, intensidade da constipação e tempo de trânsito colônico nos constipados funcionais. Conclusães - Em constipados funcionais a gravidade da constipação não depende apenas da ingestão de fibras, que, também não é a única contributiva para as variações no tempo de trânsito colônico. Este diferencia os pacientes normais dos constipados e nestes, aqueles com trânsito alterado que exigem abordagens diferentes da suplementação de fibras.

DESCRITORES - Constipação intestinal. Fibra alimentar. Trânsito gastrointestinal.

\section{INTRODUÇÃO}

A constipação não é uma doença e nem um sinal, mas um sintoma ${ }^{(9,19)}$ e, como tal, pode ser originada de vários distúrbios intestinais ou extra-intestinais ${ }^{(27)}$. Chega a atingir cerca de $50 \%$ dos atendimentos em ambulatório de clínica especializada em gastroenterologia e $14 \%$ a $15 \%$ da população em geral ${ }^{(16,22,24,42,44)}$, freqüência esta que aumenta com a idade, chegando a atingir $40 \%$ de pacientes idosos, com idade acima de 65 anos $^{(43)}$. A principal queixa dos pacientes com constipação é a diminuição da freqüência das evacuações. Entretanto, atualmente, a freqüência evacuatória, por si só, está deixando de ser importante na definição da constipação ${ }^{(46)}$. Os pacientes constipados relatam, muitas vezes, a presença de dor evacuatória, dor abdominal, fezes endurecidas, ressecadas ${ }^{(4)}$, que perderam sua forma tornandose, freqüentemente, fragmentadas, com aspecto caracterizado como coprólitos, que são eliminadas em quantidades menores que o habitual, porém em freqüência diária dentro de um padrão que pode ser considerado normal, isto é, dentro da faixa de três evacuações por dia a uma evacuação a cada 3 dias ${ }^{(15,40,41)}$. Além disso, não é incomum a persistência da sensação de evacuação incompleta após o ato evacuatório e o relato da necessidade da utilização de manobras auxiliares manuais, mecânicas ou de supositórios e enemas para a eliminação das fezes ${ }^{(15,20,38)}$.

Entre os vários tipos de constipação, destaca-se a funcional que se caracteriza pela ausência de causas orgânicas definidas, detectáveis pelos métodos de investigações atualmente disponíveis. É a forma mais comum da constipação em nosso meio. A constipação intestinal funcional, de acordo com os critérios de Roma II, é diagnosticada em pacientes que apresentam pelo menos duas das seguintes queixas, com duração de no mínimo 12 semanas, não necessariamente consecutivas, durante o último ano: a) menos de três evacuações por semana; b) fezes duras ou sensação de evacuação incompleta em pelo menos $25 \%$ das evacuações; c) dificuldade para

\footnotetext{
Trabalho realizado nos ambulatórios da Disciplina de Gastroenterologia do Departamento de Clínica Médica, no Hospital das Clínicas da Faculdade de Medicina de Botucatu, Universidade Estadual Paulista - UNESP, Botucatu, SP.

'Disciplina Metabolismo e Nutrição, Fisiopatologia e Dietoterapia, Faculdade Assis Gurgacz, Cascavel, PR.; '2Disciplina de Gastroenterologia e Nutrição, Faculdade de Medicina de Botucatu, Universidade Estadual Paulista - UNESP, Botucatu, SP.

Correspondência: Dra. Adriana Cruz Lopes - Rua João Pessoa, 107 - apt.81 - Centro - 85812-070 - Cascavel, PR. E-mail: alnutricao@yahoo.com.br
} 
evacuar em pelo menos $25 \%$ das evacuações; d) necessidade de manipulação digital para facilitar a saída das fezes ${ }^{(2)}$.

De acordo com BURKITT et al. ${ }^{(11)}$, um fator importante da gênese da constipação funcional, seria uma dieta pobre em fibras alimentares. Geralmente, seu tratamento clínico é bem sucedido com a suplementação das fibras na dieta, de forma natural ou farmacológica ${ }^{(49)}$. Problema clínico importante se estabelece quando os pacientes constipados funcionais, após corrigirem adequadamente a ingestão de fibra para 20 a $30 \mathrm{~g} /$ dia, conforme recomendação preconizada como normal $^{(28,29,48,49)}$ ou mesmo acima dela, mantêm-se constipados. Este grupo de pacientes, de difícil tratamento, tem sido alvo de estudos das funções motoras do cólon, pela determinação do tempo de trânsito colônico (TTC) $)^{(6,26)}$. As informações são poucas e controversas sobre o comportamento do TTC nos pacientes com constipação funcional $^{(13,21,51)}$, principalmente naqueles que não melhoram após a reposição adequada de fibra. Além disso, falta entendimento sobre o relacionamento entre a quantidade de ingestão das fibras com o grau da intensidade da constipação e deste com os valores do TTC ${ }^{(28,30,31)}$. Não está claro, com os dados disponíveis, se o conteúdo de fibra da dieta é fator contributivo determinante para os diferentes padrões de TTC $^{(18,49)}$.

Desse modo, este trabalho foi delineado para avaliar: 1 . se pacientes com constipação funcional, após tratamento, continuam ingerindo fibras alimentares em quantidades diferentes das pessoas normais no seu dia a dia; 2 . se o método de avaliação do TTC com marcadores radiopacos é capaz de mostrar alterações no TTC dos pacientes com constipação funcional, comparando-se com pessoas normais; 3. se a ingestão de fibras tem relações com a intensidade da constipação e com o TTC total e segmentar em pacientes com constipação funcional; e 4. se a intensidade da constipação se relaciona com TTC.

\section{MÉTODOS}

Foram incluídas no estudo 48 pessoas, sendo 30 (26 do gênero feminino e 4 do gênero masculino) com constipação funcional crônica, de acordo com os critérios de Roma II, que não tiveram sucesso após 6 meses com os tratamentos convencionais de suplementação de fibra alimentar, e 18 pessoas voluntárias normais ( 15 do gênero feminino e 3 do gênero masculino), sem queixas digestivas. Nos pacientes constipados, para a exclusão de lesões orgânicas, metabólicas e/ou endócrinas, foram realizados, após o exame clínico, exames radiológicos contrastados (enema opaco), endoscópicos, quando indicados e dosagens laboratoriais bioquímicas (glicemia), sorológicas (para doença de Chagas) e hormonais ( $\mathrm{TSH}, \mathrm{T}_{3}$ e $\mathrm{T}_{4}$ ).

O método utilizado para avaliação do TTC foi baseado naquele descrito por METCALF et. al. ${ }^{(26)}$ o qual prevê a ingestão de 20 marcadores diários, durante 3 dias consecutivos sempre no mesmo horário, com realização de $\mathrm{Rx}$ abdominal simples no $4^{\circ}$ dia. Para facilitar a análise dos cálculos, utilizou-se a ingestão de 24 marcadores diários ao invés de 20.

O TTC em um segmento (TTCs), quando se usou a ingestão de 24 marcadores de um tipo apenas, a cada $24 \mathrm{~h}$, é representado pela equação:
$\mathbf{T T C}_{\mathrm{s}}=\mathbf{n}_{\mathrm{s}}(\Delta \mathrm{T} / \mathrm{N})$ onde:

$\mathrm{n}_{\mathrm{s}}=$ número de marcadores vistos no $\mathrm{Rx}$ em determinado segmento(s) do cólon;

$\Delta \mathrm{T}=$ intervalo de tempo em horas (24), entre as consecutivas ingestões dos marcadores.

$\mathrm{N}=$ número de marcadores ingeridos em cada dia (24).

Dessa forma, o número de marcadores encontrados em determinado segmento do cólon $\left(\mathrm{n}_{\mathrm{s}}\right)$ representará o tempo de trânsito, em horas, naquele segmento, porque o resultado da divisão $(\Delta \mathrm{T} / \mathrm{N})$ é igual a 1 .

Os marcadores foram confeccionados a partir de cateteres radiopacos com 1,0 $\mathrm{mm}$ de diâmetro externo, medindo $4 \mathrm{~mm}$ de comprimento e condicionados em drágeas gelatinosas $\mathrm{n}^{\mathrm{o}}$ zero, contendo 24 marcadores em cada uma. A primeira dose (uma drágea com 24 marcadores) foi ingerida no ambulatório sob supervisão do pesquisador e as demais (dias 2 e 3 ) foram ingeridas na residência do paciente ou do voluntário.

Os segmentos do cólon foram determinados utilizando as referências ósseas no $\mathrm{Rx}$ simples de abdome, descrito por ARHAN et al. ${ }^{(6)}$, um pouco ampliadas em relação ao segmento retossigmoideano, obtendo-se os segmentos: cólon direito, cólon esquerdo e retossigmóide.

Os valores máximos normais para os tempos de trânsito, em cada segmento do cólon e para o TTC total, foram determinados utilizando-se os respectivos valores médios dos tempos obtidos em cada segmento no grupo de voluntários sadios, acrescidos de 2 desvios-padrão (média $+2 \mathrm{SD}$ ). $\mathrm{O}$ tempo total de trânsito corresponde à soma dos tempos dos três segmentos.

A intensidade da constipação foi avaliada de acordo com o estudo de $\mathrm{AGACHAN}^{(1)}$, que determinou um escore baseado nas principais queixas dos pacientes. Os sintomas incluídos para a avaliação do valor total do escore foram freqüência evacuatória, dificuldade/esforço para evacuar, dor à evacuação, sensação de evacuação incompleta, dores abdominais, tempo gasto para iniciar a evacuação, tipo de auxílio para evacuação, tentativas falhas/dia e duração da constipação. A intensidade de cada sintoma variou dentro da faixa de 0 a 4, com exceção do item tipo de auxílio para evacuação, que variou de 0 a 2 .

A constipação foi classificada em discreta quando a soma total dos valores obtidos (escore) variou de 0 a 10 , em moderada quando a soma variou de 11 a 20 e intensa quando a soma final variou de 21 a 30.

Os pacientes com constipação funcional e os voluntários foram submetidos a avaliação de ingestão alimentar de fibras, pelo método de registro alimentar de 7 dias consecutivos, incluindo fim de semana, considerando-se as dietas habituais, sem orientação específica para suplementação de fibras. As quantidades em gramas de cada alimento ingerido, foram calculadas tomandose por base as quantidades de medidas caseiras convencionais, cujos modelos padronizados foram apresentados aos participantes do estudo, registradas pelos participantes do estudo, durante os 7 dias consecutivos antes de serem submetidos ao estudo do TTC. O cálculo das quantidades de calorias, gorduras, proteínas e fibras contidas nos alimentos consumidos diariamente foi feito com o auxílio do Programa Virtual Nutri versão 1.0, desenvolvido por PHILIPPI et. al. ${ }^{(33)}$ e também por informações contidas nos rótulos dos alimentos quando estes não se encontravam referidos no banco 
de dados do Programa. As quantidades de fibras ingeridas pelos pacientes e voluntários são expressas pela média da ingestão dos 7 dias avaliados. Foram padronizados como normal a ingestão de 20 a 35 $\mathrm{g} /$ dia, conforme recomendado pela American Dietetic Association ${ }^{(33)}$. A ingestão de fibra também foi relacionada à ingestão de calorias para diminuir o peso da quantidade de alimentos ingeridos no total pelos pacientes constipados e pelos voluntários.

As diferenças das proporções dos gêneros entre os grupos foram analisadas pelo teste de Fischer. As comparações das diferenças das demais variáveis entre os grupos foram analisadas pelo teste estatístico de Mann-Whitney ${ }^{(32)}$. As associações entre ingestão de fibra, intensidade da constipação e TTC total e segmentar foram feitas utilizando-se o coeficiente de correlação de Sperman ${ }^{(32)}$.

Todas as discussões foram realizadas no nível de 5\% de significância. Os dados são apresentados na forma das médias com seus respectivos desvios-padrão.

\section{RESULTADOS}

As diferenças das idades entre os grupos não se mostraram significantes estatisticamente $(44,5 \pm 15,0$ constipados $\mathrm{x}$ 45,9 $\pm 14,3$ controles; $P>0,05$ ), bem como a distribuição entre os sexos, porém houve predominância do sexo feminino $(85,4 \%$ constipados x 83,3\% controles; $P=0,84$ ) em ambos.

Os pacientes constipados apresentaram maior ingestão de fibras que os voluntários sadios, avaliadas tanto em gramas/dia (26,3 $\pm 12,9$ $\mathrm{g} / \mathrm{d}$ constipados $\mathrm{x} 9,3 \pm 5,2 \mathrm{~g} / \mathrm{d}$ controles; $P<0,001$ ), quanto em g/1000 $\mathrm{kcal} / \mathrm{dia}(12,3 \pm 7,6 \mathrm{~g} / 1000 \mathrm{kcal}$ constipados x 6,1 $\pm 1,9 \mathrm{~g} / 1000 \mathrm{kcal}$ controles; $P<0,001)$. Entre os pacientes constipados, $23,3 \%$ apresentaram ingestão de fibra superior ao preconizado, $36,7 \%$ apresentaram ingestão dentro da faixa normal e em $40 \%$ deles a ingestão de fibra foi inferior ao recomendado. No grupo de voluntários sadios apenas um paciente $(3,3 \%)$ apresentou ingestão de fibras de acordo com a recomendação, o restante apresentou ingestão abaixo da preconizada.

A classificação da intensidade da constipação no grupo de constipados atingiu níveis de moderada (10 pacientes) a intensa (19 pacientes), com escore médio de 21,3 , com desvio-padrão de 4,07. Apenas um paciente constipado apresentou valor do escore dentro da faixa de constipação discreta. Não houve correlação significante entre a ingestão de fibra e a intensidade da constipação, como se observa na Figura 1.

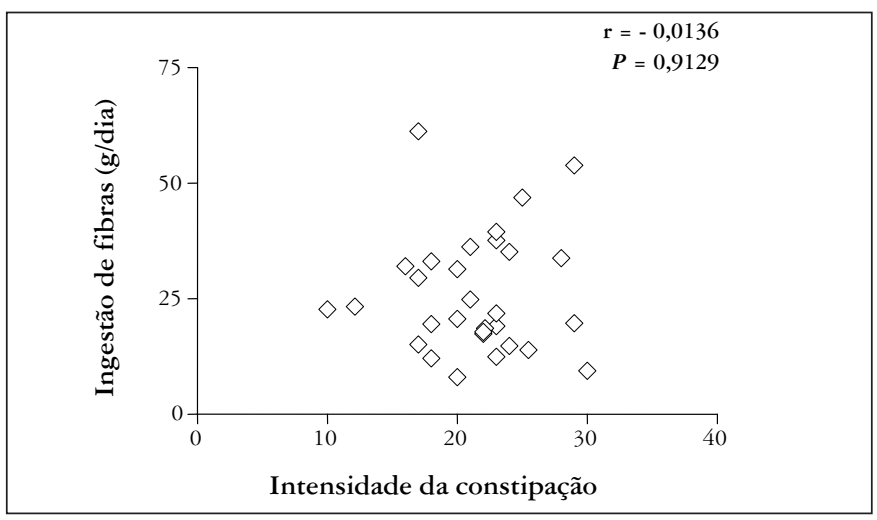

FIGURA 1. Correlação entre ingestão de fibra e intensidade da constipação no grupo de pacientes constipados
Os valores máximos normais obtidos para os tempos de trânsito foram: $15,4 \mathrm{~h}, 34,8 \mathrm{~h}$. 17,6h e $58,8 \mathrm{~h}$ para o trânsito no cólon direito, no cólon esquerdo, no segmento retossigmoideano e trânsito no cólon total, respectivamente.

A análise do comportamento do TTC entre os grupos, mostrou que o grupo de constipados apresentou, em média, tempo de trânsito total significantemente maior que o grupo controle, principalmente em razão dos maiores tempos no cólon direito e região retossigmoideana (Tabela 1). Dentro do grupo constipados, o TTC total foi normal $(<58,8 \mathrm{~h})$ em 20 pacientes $(66,6 \%)$. Entre estes, foram observados trânsitos lentos em segmentos isolados do cólon: no cólon direito (nove pacientes), no cólon esquerdo (três pacientes) e na região retossigmoideana (oito pacientes). Os outros 10 pacientes $(33,3 \%)$ do grupo constipados mostraram o TTC total acima do normal $(>58,8)$. Entre estes, oito $(80 \%)$ apresentaram padrão de inércia colônica (retenção homogênea dos marcadores radiológicos em todos os segmentos do cólon), um $(10 \%)$ apresentou padrão de estase isolada do cólon esquerdo e um outro (10\%) apresentou padrão de obstrução de saída (retenção dos marcadores radiológicos quase exclusivamente no segmento retossigmoideano do cólon).

TABELA 1. Tempo de trânsito colônico total e segmentar nos pacientes constipados e voluntários. Estão representados os tempos médios, em horas, com seus desvios-padrão, e os respectivos resultados dos testes estatísticos de Mann-Whitney

\begin{tabular}{lcccc}
\hline Grupos & TTCd & TTCe & TTRS & TTCT \\
\hline GCF & $12,5 \pm 10,7 \mathrm{~h}$ & $15,8 \pm 10,8 \mathrm{~h}$ & $15,8 \pm 10,8 \mathrm{~h}$ & $41,0 \pm 22,8 \mathrm{~h}$ \\
GVS & $5,6 \pm 4,9 \mathrm{~h}$ & $10,8 \pm 12,0 \mathrm{~h}$ & $5,4 \pm 6,1 \mathrm{~h}$ & $21,8 \pm 18,5 \mathrm{~h}$ \\
Estatística & $P<0,05$ & $P>0,05$ & $P<0,05$ & $P<0,005$ \\
\hline
\end{tabular}

TTCT= tempo de trânsito colônico total; $\Pi \mathrm{TC}=$ tempo de trânsito no cólon direito; $\Pi \mathrm{TC}=$ tempo de trânsito no cólon esquerdo; TTRS = tempo de trânsito no segmento retossigmoideano

No grupo de voluntários normais, todos apresentaram TTC total dentro dos padrões da normalidade. Houve um paciente com tempo de trânsito lento no cólon direito e outro na região retossigmoideana, lentificações que não foram suficientes para aumentar seus tempos de trânsito colônico total.

Não se observaram correlações estatisticamente significantes entre a ingestão de fibra e o TTC total e segmentar tanto no grupo constipado como no grupo controle. Também não foram encontradas correlações significantes entre a intensidade da constipação e o TTC no grupo constipado.

\section{DISCUSSÃO}

No grupo com constipação funcional, houve predomínio de mulheres jovens confirmando os dados da literatura ${ }^{(10,11,41)}$ que apontam maior freqüência deste diagnóstico em pessoas jovens do sexo feminino. A aplicação do escore de intensidade neste grupo, mostrou valores que expressam a presença do quadro de constipação intestinal de moderada a severa. Portanto, são pacientes que se sentem bastante importunados com a presença desse sintoma, apesar de ingerirem, na sua maioria, fibra na dieta habitual em quantidade igual ou acima do recomendado. Esses pacientes constipados funcionais não foram sensíveis às suplementações de fibra na dieta, fato que se confirma pela ausência de correlações significativas entre as 
quantidades de fibras ingeridas e a intensidade da constipação encontradas neste grupo. Confirma-se, dessa forma, a existência de pacientes com constipação funcional que não respondem à ingestão de fibra alimentar. Esses pacientes precisam ser investigados mais cuidadosamente no sentido de se excluir causas de constipação não detectáveis pelos procedimentos usuais de investigação. Atualmente, tem se destacado pela sua utilização crescente, a avaliação do TTC total e segmentar por meio de marcadores radiológicos. A aplicação desse procedimento, após as padronizações necessárias para a determinação dos valores normais, permitiu diferenciar claramente os pacientes constipados dos normais do grupo controle. $\mathrm{O}$ grupo controle apresentou TTC menor em relação aos observados por outros autores e, em conseqüência disto, os valores máximos normais dos TTC total e segmentar obtidos neste trabalho, foram menores que os citados na literatura, principalmente para o cólon direito, retossigmóide e trânsito total ${ }^{(6,13,22,26,39,51)}$. Os pacientes constipados apresentaram, em média, TTC total quase duas vezes maior que o do grupo controle. Entretanto, considerando-se como máximo normal o valor de 58,8h para o TTC total, os valores obtidos em média no grupo de pacientes constipados não ultrapassaram esse valor. Porém, 10 pacientes constipados (33\%) apresentaram TTC total acima desse valor normal, caracterizando um grupo de pacientes constipados com trânsito lento. Os outro 20 pacientes constipados apresentaram TTC total normal, caracterizando outro grupo de constipados com tempo de trânsito normal. Esses achados não diferem dos encontrados por outros autores que utilizaram metodologia semelhante, em pacientes constipados funcionais ${ }^{(5,}$ 18, 23,24,35). No grupo de constipados com trânsito lento foi possível caracterizar a presença da inércia colônica e da constipação de saída. $\mathrm{Na}$ inércia colônica têm sido relatadas lesões do plexo nervoso mioentérico ou mesmo da musculatura entérica ${ }^{(7,8,47)}$, ainda de difícil entendimento e diagnóstico, produzindo em conseqüência, diminuição da sensibilidade do cólon aos reflexos motores ${ }^{(37)}$, com lentificação ou mesmo parada da propulsão da massa fecal, com estase da mesma em todos os segmentos do cólon ${ }^{(36)}$.

Na constipação de saída ocorre retenção de fezes no segmento retossigmoideano por mecanismos obstrutivos mecânicos, na maioria dos $\operatorname{casos}^{(4)}$, excetuando-se destes a ocorrência do anismo. Neste, a retenção de fezes se deve à evacuação imperfeita do reto por contrações paradoxais no ato evacuatório, do músculo púbis retal, do assoalho pélvico e dos esfíncteres retais, cujas causas ainda não estão bem estabelecidas ${ }^{(12)}$. Pacientes com essas alterações orgânicas (inércia colônica e anismo) não devem ser considerados como acometidos de constipação funcional. É por esta razão que respondem mal ao emprego de fibra em seu tratamento, alguns necessitando até mesmo de tratamento cirúrgico. Pela mesma razão, pacientes com anismo devem ser submetidos a tratamentos específicos baseados em treinamentos evacuatórios denominados "biofeedback"(4). Assim, fica evidente, como apontam os resultados do presente estudo, que a população dos pacientes constipados funcionais crônicos é constituída por indivíduos com múltiplas causas para sua constipação. Não basta apenas diagnosticá-los conforme os critérios de Roma II, como também insistir na suplementação da fibras em pacientes que não respondem ao tratamento.
Nestes, é necessário avançar na investigação com métodos apropriados para separar os com constipação funcional daqueles com patologias orgânicas bem definidas.

Em todos os pacientes do grupo de constipados com trânsito normal, bem como em dois pacientes dentro do grupo de voluntários sadios, foram detectados tempos de trânsito aumentados em segmentos isolados do cólon. $\mathrm{O}$ aumento do tempo de trânsito em determinado segmento isolado do cólon não foi suficiente para aumentar o TTC total nesses pacientes. Fica a dúvida se essas alterações do tempo de trânsito em segmentos isolados do cólon se relacionam com as queixas dos pacientes constipados com tempo de trânsito normal. Os significados desses achados aguardam mais estudos.

BURKITT et al. ${ }^{(11)}$ descreveram a relação entre constipação e hábitos alimentares, demonstrando que o consumo de maior quantidade de fibra se associa ao tempo de trânsito intestinal mais rápido em pessoas normais. Este fato não foi constatado na presente série, pois não se observou nenhuma correlação entre a ingestão de fibras e o TTC total e segmentar tanto no grupo controle como no de constipados. O mesmo aconteceu quando se avaliaram as relações entre esses parâmetros no conjunto de todos os pacientes, independentemente dos grupos. Esses achados assemelham-se aos descritos por DANQUECHIN et al. ${ }^{(14)}$.

A ingestão de fibras pelos voluntários normais foi baixa e todos se apresentaram sem queixas digestivas. É grande o número de pessoas que ingerem quantidades de fibras abaixo do preconizado e, mesmo assim, não apresentam sintomas de constipação ${ }^{(17,25)}$. Ainda não está totalmente esclarecido se a proporção entre os nutrientes das refeições afeta as respostas motoras do cólon. Entretanto, foi demonstrado que dietas ricas em carboidratos induzem à resposta motora do cólon, embora em períodos de curta duração, quando comparadas com as dietas ricas em gorduras que também induzem à atividade fásica e segmentar do cólon. Além disso, os efeitos motores das gorduras podem ser modificados pela sua combinação com proteínas e carboidratos ${ }^{(34,50)}$. Os controles voluntários apresentaram ingestão de fibra por 1.000 calorias muito menor em relação aos constipados, mostrando que os controles tiveram um aporte maior de calorias por grama de fibra ingerida. Assim, é perfeitamente possível supor que a falta de fibras nas dietas dos controles tenha sido compensada, em termos de estímulo da atividade colônica, por maiores quantidades de carboidratos e gorduras.

\section{CONCLUSÃO}

Não houve relação entre a ingestão de fibras, o TTC total e segmentar e a intensidade de constipação nos pacientes constipados. Estes dados mostram que a intensidade da constipação não depende apenas da ingestão de fibras, não é contributiva para as variações no tempo de trânsito colônico. Este, entretanto, diferencia os pacientes normais dos constipados e nestes, os com trânsito alterado que exigem abordagens diferentes da suplementação de fibras.

A importância da lentificação em segmento isolado do cólon na constipação, precisa ser melhor avaliada. 
Lopes AC, Victoria CR. Fiber intake and colonic transit time in functional constipated patients. Arq Gastroenterol. 2008;45(1):58-63.

ABSTRACT - Background - Patients with functional constipation presenting no response to treatment using fibers supplement represents important clinical issue. Aims - To evaluate the relations among the amount of ingested fiber, the constipation intensity and the colonic transit time in patients with functional constipation. Methods - We evaluated 30 patients, presenting no response to treatment using fibers supplement, and 18 healthy volunteers conducting individual inquiry into fibers intake, constipation intensity and the total and segmental colonic transit evaluation using radiopaque markers. Results - In the constipated, despite the good level of fiber intake $(26.3 \pm 12.9 \mathrm{~g}$, constipated x $9.3 \pm 5,2 \mathrm{~g}$, control), the symptoms of constipation was serious (score $=21.3 \pm$ 4.07). Mean total colonic transit was $58.8 \mathrm{~h}$. The colonic transit was slower in the constipated group ( $41.0 \pm 22.8$ hours, constipated x $21.8 \pm 18.5 \mathrm{~h}$, control). In constipated patients with slow colonic transit ( $>58.8 \mathrm{~h})$ there were colonic inertia (eight), outlet constipation (one) and slow transit in left colon (one), and among constipated patients with normal colonic transit $(<58.8 \mathrm{~h})$, there were isolated slow transit, in the right colon (nine), left colon (three) and in the rectosigmoid segment (eight). There were no relation among the amount of ingested fiber, constipation intensity and the colon transit. Conclusions - In the functional constipation the gravity of symptoms does not depend only on the dietary fibers intake, which is not the only responsible for the differences in the colonic transit. The colonic transit can differentiate normal from constipated patients and, among them, those with altered transit that demand approaches distinct of fiber supplementation.

HEADINGS - Constipation. Dietary fiber. Gastrointestinal transit.

\section{REFERÊNCIAS}

1. Agachan F, Chent T, Pfeifer J, Reisseman P, Wexner DAS. Constipation scoring system to simplify evaluation and management of constipated patients. Dis Colon Rectum. 1996;39:681-5

2. Ambrogini OJ, Miszputen SJ. Constipação intestinal crônica. Rev Bras Med 2002;59:133-9.

3. American Dietetic Association. Position of the American Dietetic Association: health implications of dietary fiber. Am Diet Assoc. 1988;88:217-21.

4. Andromanakos N, Skandalakis P, Troupis T, Filippou D. Constipation of anorectal outlet obstruction: pathophysiology, evaluation and management. J Gastroenterol Hepatol. 2006;21:638-46.

5. Ansari R, Sohrabi MR, Roohi S, Mikaeli J, Massarrat S, Mehrizi ST, Malekzadeh R. Colonic transit time in 64 Iranian patients with idiopathic chronic constipation. Arch Iran Med. 2001;4:5-9.

6. Arhan P, Devroede G, Jehannin B, Lanza M, Faverdin C, Dornic C, Persoz B, Tétreault L, Perey B, Pellerin D. Segmental colonic transit time. Dis Colon Rectum. 1981;24:625-9.

7. Bassotti G, Villanacci V. Slow transit constipation: a functional disorder becomes an enteric neuropathy. World J Gastroenterol. 2006;12:4609-13.

8. Bassotti G, Villanacci V, Cathomas G, Maurer CA, Fisogni S, Cadei M, Baron L, Morelli A, Valloncini E, Salerni B. Enteric neuropathology of terminal ileum in patients with intractable slow-transit constipation. Hum Pathol. 2006;37:1252-8.

9. Bleijenberg G, Kuijpers HC. Biofeedback treatment of constipation: a comparison of two methods. Am J Gastroenterol. 1994;89:1021-6.

10. Briejer MR, Schuurkes JA, Sarna SK. Idiopathic constipation: too few stools and too little knowledge. Trends Pharmacol Sci. 1999;20:1-3.

11. Burkitt DP, Walker AR, Painter NS. Effect of dietary fiber of stools and transit times and its role in the causation of disease. Lancet. 1972;2:1408-12.

12. Caruso R, Olza MT, Azcárate H, Arrieche MT, Pestana E, Morera C, Jaen D. Evaluación manométrica de los parâmetros voluntários en pacientes estenidos con anismo. GEN. 2004;58:13-5.

13. Chaussade S, Khyari A, Roche H, Garret M, Gaudric M, Couturier D, Guerre J. Determination of total and segmental colonic transit time in constipated patients. Results in 91 patients with a new simplified method. Dig Dis Sci. 1989;34:1168-72.

14. Danquechin Dorval E, Barbieux JP, Picon L, Alison D, Codjovi P, Rouleau P. Simplified measurement of colonic transit time by one radiography of the abdomen and a single type of marker. Normal values in 82 volunteers related to the sexes. Gastroenterol Clin Biol. 1994;18:141-4.

15. Everhart JE, Vay Liang W Go, Johannes RS, Stacey CF, Harold PR, Lon RW. A longitudinal survey of self-reported bowel habits in the United States. Dig Dis Sci. 1989;34:1153-62.

16. Ferguson A, Sircus W, Eastwood MA. Frequency of functional gastrointestinal disorders. Lancet. 1977;2:613-4.

17. Galvin MA, Kiely M, Harrinton KE, Robson PJ, Moore R, Flynn A. The North/South Ireland food consumption survey: the dietary fiber intake of Irish adults. Public Health Nutr. 2001;4:1061-8.

18. Guimarães EV, Goulart EMA, Penna FJ. Dietary fiber intake, stool frequency and colonic transit time in chronic functional contipation in children. Braz J Med Biol Res. 2001;34:1147-53.
19. Han C, Kuijpers MD. Application of the colorectal laboratory in diagnosis and treatment of functional constipation. Dis Colon Rectum. 1990;33:35-9.

20. Heaton KW, Cripps HA. Straining at stool and laxative taking in an English population. Dig Dis Sci. 1993;38:1004-8.

21. Husni-Hag-Ali R, Gómez Rodriguez BJ, Mendonza Olivares FJ, García Montes JM, Sáchez-Gey Venegas S, Herrerías Gutierrez JM. Measuring colonic transit time in chronic idiopathic constipation. Rev Esp Enferm Dig. 2003;95:181-90.

22. Jorge JMN, Habr-Gama A. Tempo de trânsito colônico total e segmentar: análise crítica dos métodos e estudo de indivíduos normais com marcadores radiopacos. Rev Bras Coloproctol. 1991;11:55-60.

23. Krevsky B, Maurer AH, Fisher RS. Patterns of colonic transit in chronic idiopathic constipation. Am J Gastroenterol. 1989;84:127-32.

24. Krishnamurthy S, Schuffler MD, Rohrmann CA, Pope CE 2nd. Severe idiopathic constipation is associated with a distinctive abnormality of the colonic myenteric plexus. Gastroenterology. 1985;88:1:26-34.

25. Mattos LL, Martins IS. Dietary fiber consumption in an adult population. J Public Health. 2000;34:50-5

26. Metcalf AM, Phillips SF, Zinsmeister AR, Maccarty RL, Beart RW, Wolff BG. Simplified assessment of segmental colonic transit. Gastroenterology. 1987;92:40-7.

27. Mollen RM, Claassen AT, Kuijpers JH. The evaluation and treatment of functional constipation. Scand J Gastroenterol. 1997;223:8-17.

28. Morais MB, Vitolo MR, Aguirre AN, Medeiros EHGR, Antonelli EMAL, FagundesNeto U. Teor de fibra alimentar e de outros nutrientes na dieta de crianças com e sem constipação crônica funcional. Arq Gastroenterol. 1996;33:93-101.

29. Morais MB, Vitolo MR, Aguirre AN, Fagundes-Neto U. Measurement of low dietary fiber intake as a risk factor for chronic constipation in children. J Pediatr Gastroenterol Nutr. 1999;29:132-5.

30. Müller-Lissner SA. The effect of wheat bran on stool weight and gastrointestinal transit time. A meta-analysis. Br Med J. 1988;296:615-7.

31. Müller-Lissner SA, Kamm MA, Scarpignato C, Wald A. Myths and misconceptions about chronic constipation. Am J Gastroenterol. 2005;100:232-42.

32. Norman GR, Streiner DL. Biostatistics: the bare essentials. St Louis: Mosby; c1994. p.26.

33. Philippi ST, Szarfarc SC, Latterza AR. Virtual Nutri [programa de computador]. Versão 1.0 para Windows. São Paulo: Departamento de Nutrição de Saúde Pública da Universidade de São Paulo, 1999.

34. Rao SS, Kavelock R, Beaty J, Ackerson K, Stumbo P. Effects of fat and carbohydrate meals on colonic motor response. Gut. 2000;46:205-11.

35. Rolim AS. Contribuição do estudo do tempo de trânsito colônico e da videodefecografia na constipação intestinal essencial [dissertação]. São Paulo: Faculdade de Medicina da Universidade de São Paulo; 2001.

36. Shafik A, Ahmed I. Study of the motile activity of colon in rectal inertia constipation. J Gastroenterol Hepatol. 2002;17:270-5.

37. Shafik A, Shafik AA, El-Sibai O, Mostafa RM. Electric activity of the colon in subjects with constipation due to total colonic inertia: an electrophysiologic study. Arch Surg. 2003; 138:1007-11.

38. Sandler RS, Jordan MC, Shelton BJ. Demographic and dietary determinants of constipation in the US population. Am J Public Health. 1990;80:185-9.

39. Sobrado CW, Pires CEF, Habr-Gama A, Kiss DR. Avaliação do tempo de trânsito colônico com marcadores radiopacos. Estudo com voluntários assintomáticos. Rev Col Bras Cir. 2005;32:111-4 
40. Soffer EE. Constipation: an approach to diagnosis, treatment, referral. Cleve Clin J Med. 1999;66:41-6.

41. Sweeney M. Constipation: diagnosis and treatment. Home Care Provid. 1997;2:250-5.

42. Switz DM. What the gastroenterologist does all day. A severy of a state society's practice. Gastroenterology. 1976;70:1048-50.

43. Talley NJ, Fleming KC, Evans JM, O'Keefe EA, Weaver AL, Zinsmeister AR, Melton LJ 3rd. Constipation in an elderly community: a study of prevalence and potential risk factors. Am J Gastroenterol. 1996;91:19-25.

44. Thompson WG, Heaton KW. Functional bowel disorders in apparently healthy people. Gastroenterology. 1980;79:283-8

45. Thompson WG, Longstreth GF, Drossman DA, Heaton KW, Irvine EJ, MullerLissner SA. Functional bowel disorders and functional abdominal pain. 1999;45 (Suppl 2):II43-7

46. Wald A. Constipation. Med Clin North Am. 2000;84:1231-46.

47. Wedel T, Van Eys GJ, Waltregny D, Glénisson W, Castronovo V, Vanderwinden JM. Novel smooth muscle markers reveal abnormalities of the intestinal musculature in severe colorectal motility disorders. Neurogastroenterol Motil. 2006;18:526-38.
48. Williams CL, Bollella M, Wynder ELA. A new recommendation for dietary fiber in childhood. Pediatrics. 1995;96(5Pt2):985-8.

49. Winfried A, Voderholzer MD, Winfried Schatke MD, Birgit E, Mühldorfer Andreas G, Klauser MD, Berndt Birkner MD, Stefan A, Müller-Lissner MD. Clinical response to dietary fiber treatment of chronic constipation. Am J Gastroenterol. 1997;92:95-8.

50. Zalar A, Riachi G, Heresbach D, Guédon C, Denis P, Ducrotté P. Influence de la charge lipidique du repas sur les réponses motrices duodeno-jejunale et sigmoïdienne chez le volontaire sain. Gastroenterol Clin Biol. 1996;20:2-7.

51. Zaslavsky C, Silveira TR, Maguilnik I. Total and segmental transit time with radiopaque markers in adolescents with functional constipation. J Pediatr Gastroenterol Nutr. 1998;27:138-42
Recebido em 10/10/2005 Reapresentado em 11/7/2007. Aprovado em 19/10/2007. 